\title{
Logistical Causes of Food Waste: A Case Study of a Norwegian Distribution Chain of Chilled Food Products
}

\author{
Lukas Chabada $^{1}$, Cecilie Maria Damgaard ${ }^{2}$, Heidi Carin Dreyer ${ }^{1}$, \\ Hans-Henrik Hvolby ${ }^{2,1}$, and Iskra Dukovska-Popovska ${ }^{2}$ \\ ${ }^{1}$ Norwegian University of Science and Technology, \\ Department of Production and Quality Engineering, Trondheim, Norway \\ \{lukas.chabada, heidi.c.dreyer\}@ntnu.no \\ ${ }^{2}$ Aalborg University, Centre for Logistics, \\ Department of Mechanical and Manufacturing Engineering, Aalborg, Denmark \\ \{cecilie,hhh\}@celog.dk, iskra@m-tech.aau.dk
}

\begin{abstract}
This study discusses logistical planning and handling activities contributing to food waste in the food distribution chain of chilled products with fixed shelf life and with an age dependent deterioration rate. The study has exploratory character and all the findings are based on the case study investigation from six Norwegian companies. The causes of food waste as identified in the case companies are grouped into four areas, namely planning decisions, data utilisation, execution of plan, and damaged products. Quantitative data indicates that for chilled products with fixed shelf life, logistical planning seems to have higher impact on financial losses from food waste than physical handling.
\end{abstract}

Keywords: logistics, causes, food waste, distribution chain, chilled products.

\section{Introduction}

Managing the physical flow of chilled products with short shelf life and sensitiveness to physical handling is challenging for the actors in the food distribution chain (FDC). The FDC includes the wholesalers, stores and logistical and transport provides which takes part in the delivering of food to end customers. The main priorities are to make sure of short lead times and sufficient conditions during the physical distribution. In the food distribution chain products are being wasted stages (Beretta et al., 2012; Eriksson et al., 2012). A study by Hanssen et al. (2013) revealed food waste of around $5-8 \%$ for chilled products at the retail stage in Norway. Such waste has economic, environmental and social impacts (Mena et al., 2011). Therefore, managers have an intention to reduce food waste. However, methods and concepts systematically approaching food waste reduction are scarce (Garrone et al., 2014). This might be because food waste related costs have been undervalued or hidden (Mena et al., 2011).

Identifying the causes of food waste is the first step towards food waste reduction. Studies by Mena et al. (2011), Beretta et al. (2012) or Muller (2013) have indicated several causes of food waste. For example, as food companies strive for a high service 
level under varying demand, they often maintain too high inventories and safety stock levels, which subsequently lead to food waste. For food products, due to its perishable nature and restricted shelf life, it is significantly important that supply and demand is aligned and that the planning and control mechanisms are designed according the characteristics of the product, market and process involved. Until now, research on food waste has mainly focused on quantifying volumes, where in the food chain waste is created and what product category which most frequently is being created. The planning and control of the FDC and the effects on food waste is still area which needs to be explored in order to understand how food waste could be reduced.

The aim of this study is to explore logistical causes of food waste in the FDC of two types of chilled products; products with fixed shelf life and products with age dependent deterioration rates. The reasons for differentiating the products are the different planning and handling practices used for the above mentioned types, as similarly used by Bakker et al. (2012). The scope of this study is on food distribution chains in Norway, whose geography is characterized by long transportation distances, scattered population centers, and challenging climatic conditions which increase lead time and lead time variability (Romsdal et al., 2011). Since the phenomenon under study is relatively new, a case study research has been selected (Yin, 2009).

Current research identifying causes of food waste is described in the section 2 followed by section describing methods of data collection and analysis. After that case description, findings and discussion on identified challenges in logistical planning and physical handling captured from interviews in case companies are presented. In the last chapter, several concluding comments are made and future work is proposed.

\section{Connecting Logistics and Food Waste}

The main reasons for food being wasted is insufficient quality and expired date (Mena et al., 2011). Setting this in relation to logistics, it can refer to time and quality which are among the main performance indicators of logistics (Shapiro et al., 1985). According to APICS (2013) logistics system consists of planning and coordination of the physical movement aspects of a firm's operations such that a flow of raw materials, parts, and finished goods is achieved in a manner that minimizes total costs for the levels of service desired. Model of food waste have been recently presented by Garrone et al. (2014) where they considered different management practices handling surplus food to define food waste from environmental, zootechnical and social perspectives. Inspired by their study and food waste definitions used by Beretta et al. (2012) or Gustavsson et al. (2011) our understanding of food waste in this study is: finished food products that are manufactured, wholesaled and retailed but not sold to the intended customer or which are sold but to a price lower than the intended price due to insufficient quality or short remaining shelf life. For example discounts due to short remaining shelf life are considered as waste. The intended customer for the wholesaler is the store and for the store the intended customer is the paying consumer.

For in-depth analysis of food waste and its logistical causes a need for locating food waste in the processes of the FDC has been recognized. Eriksson et al. (2012) 


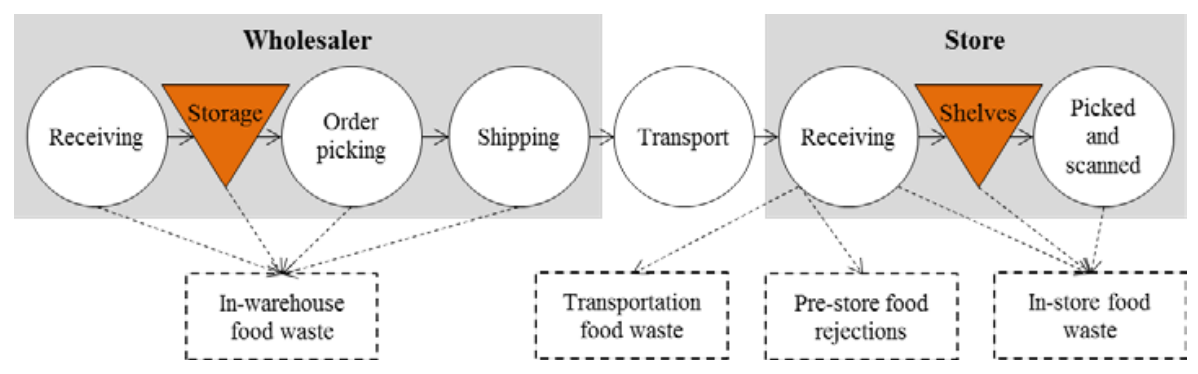

Fig. 1. Categories of food waste in the food distribution chain

defined two main types of food waste in the stores on the process level, namely in-store and pre-store food waste. This study extends on their work and adds transportation and in-warehouse food waste to the analysis (see Fig. 1).

In-warehouse food waste includes all food products wasted during the processes in the warehouse of the wholesaler. Transportation food waste includes the food waste created during transportation between wholesaler and stores. Pre-store food rejections include food products rejected by the store within 24 hours after delivery. After this period it is considered as in-store food waste. This waste belongs to the supplier in accounting terms, but usually becomes physical waste at the store (Eriksson et al., 2012). Finally, in-store food waste includes all products wasted inside the store.

The number of studies identifying the causes of food waste at different stages of the food supply chain in different countries has increased during the last couple of years. Mena, Adenso-Diaz, and Yurt (2011) have looked at the causes of food waste in the supplier-retailer interface at British and Spanish companies. They categorized the causes into mega-trends, natural constraints and management root causes where only the latter can be fully under the control of the managers, and includes issues such as inaccurate planning and forecasting, poor information sharing, poor ordering, poor handling or poor promotions management. Similarly, Muller (2013) presented a root cause analysis of food waste in Norwegian food supply chains. In that study, overproduction and over-ordering, large distribution packages, high inventory levels, inappropriate stock rotation or inadequate handling have been among the mentioned logistical causes of food waste. Finally, Beretta et al. (2013) presented causes of food waste considering the whole food supply chain in Switzerland, and categorized the causes into avoidable, possibly avoidable and unavoidable considering. They mention overproduction or high stock levels among the avoidable causes.

All of the above mentioned studies indicate that food waste could be reduced by better planning and handling of logistical activities. Since the current studies present mainly qualitative data of different causes on quite an aggregated level one can only assume, based on the frequency of evidence, that planning has a greater impact on food waste than physical handling. Moreover, the studies indicate that physical handling, decisions made by planners, and data used during the planning process could have an impact on food waste. This study investigates for the evidence of food waste causes in the case companies that can support these hypotheses. 


\section{Methods for Data Collection and Analysis}

Investigation of logistical causes of food waste is still an unexplored area, therefore, this study undertook the case study approach outlined by Yin (2009). Case companies have been selected based on their relatively high impact in the Norwegian market.

Different quantitative and qualitative data has been collected to identify causes of food waste in the case companies. Quantitative data showing the redistribution of the main causes has been collected for in-warehouse food waste and pre-store food rejections since the wholesaler is the only company that registers them. The in-house ERP system and the internal documents of the wholesaler have been used for this purpose. Since the data is registered differently in pieces, boxes and kilos depending on the product type, financial losses have been used to compare the relative values of registered causes. Quantitative data aggregates amounts from a 12 -month period and consider food waste as defined by the case companies and not as defined in the section 2 . The impact of different causes has been analyzed in relation to the total food waste.

Second, qualitative data supplements the quantitative findings by discussing underlying causes of the main causes identified in the case companies. The data has been collected via semi-structured interviews, mapping and personal observations of planning and handling processes. Causes of transportation and in-store food waste have been collected via interviews and observations at two transporters and three stores. In order to highlight the main problematic areas the identified underlying causes of food waste were grouped using the fishbone diagram (Stevenson et al., 2007).

The scope of the investigation is on chilled products, as these have been identified as the main wasted category (Gustavsson et al., 2011). This study includes product groups like dairy, meat, fish, fruit and vegetable products $(\mathrm{F} \& \mathrm{~V})$ and the main focus is on the planning and handling causes of food waste in the FDC. During the study it has been recognized that planning and handling practices of products with fixed shelf life varies from products with age dependent deterioration rate, therefore this study differentiates between these two categories when discussing causes of food waste. Products with fixed shelf life are wasted when the due date is exceeded or close to be exceeded. Besides F\&V, majority of chilled products have fixed shelf life. Products with age dependent deterioration rate are wasted when the quality is perceived to be insufficient. The majority of $\mathrm{F} \& \mathrm{~V}$ have an age dependent deterioration rate.

\section{$4 \quad$ Case Description and Findings}

The wholesaler is a major wholesaler in Norway distributing around 5500 products. Three stores are direct customers of the wholesaler consisting of one store with low and two stores with high turnover. Together with two transportation companies delivering food products between the wholesaler and the stores the companies are part of the same supply chain. First analysis of data indicates $90 \%$ of food waste in this FDC comes from wasted chilled products. Two main causes of food waste are registered at the wholesaler, namely insufficient quality and short remaining shelf life. Quantitative data on causes of food waste revealed that in two thirds of cases of in-warehouse food 
waste of products with fixed shelf life, short remaining shelf life is the main cause leaving one third for damaged products. In more than $90 \%$ of cases the main cause of in-warehouse food waste for products with age dependent deterioration rate is insufficient quality (this information comes from discussions with planners and operators in the warehouse). Similar findings can be observed for pre-store food rejections where $96 \%$ of products with age dependent deterioration rate have been rejected by stores due to insufficient quality while $64 \%$ of products with fixed shelf life have been rejected due to short remaining shelf life (see Fig. 2).

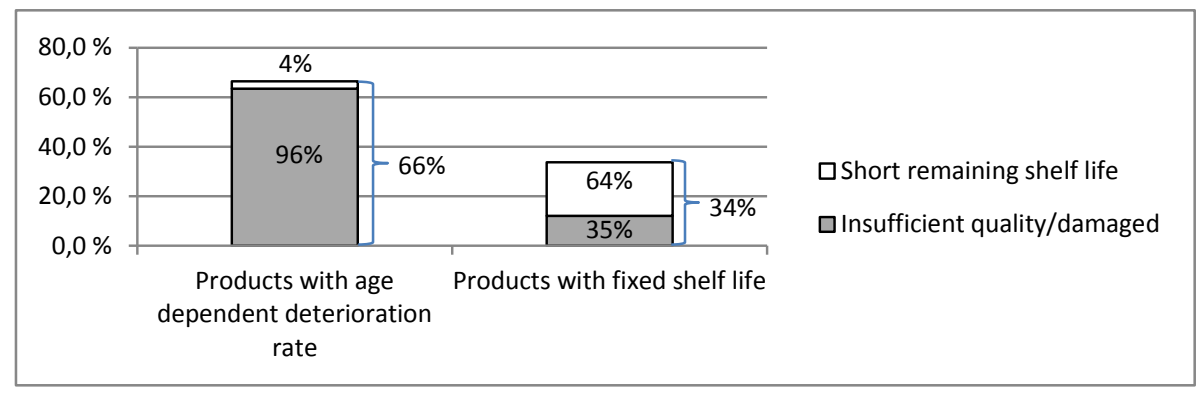

Fig. 2. Registered causes of pre-store food rejections

Fig. 2 also illustrates that around two thirds of food waste come from products with age dependent deterioration rate. This might be due to several reasons like different biological deterioration rate of products, higher margin or different planning and handling procedures of products with age dependent deterioration rate and fixed shelf life. Analysis of causes of food waste registered at the wholesaler revealed that "insufficient quality" represents mainly handling failures and "short remaining shelf life" represents mainly planning failure when considering food products with fixed shelf life. Based on this, logistical planning seems to have higher impact on financial losses than physical handling of products with fixed shelf life.

\section{$5 \quad$ Causes of Insufficient Quality and Short Remaining Shelf Life}

Company visits and interviews with planners and operators confirmed the existence of food waste categories defined in Fig. 1. Moreover, several underlying causes of insufficient quality and short remaining shelf life have been revealed. These are discussed in this chapter per food waste category, supported by previous studies when relevant.

In-warehouse food waste of products with fixed shelf life is mainly created due to short remaining shelf life. As the potential underlying causes have been pointed high inventory and safety stock levels, and quite aggregated planning and coordination of promotions with stores. For promotions the stock levels are set high in order to prevent stock outs and because demand is unpredictable, thus increasing food waste. Food waste is also increased due to product cannibalization (Taylor et al., 2009). 
Ordered amounts are sometimes increased also due to low ordering frequency and big batch sizes, when the wholesaler orders more than is needed to fit the batch size. Increased uncertainty due to promotion planning or ordering frequency has been identified also by Mena et al. (2011). More frequent deliveries and information exchange could thus reduce food waste (Kaipia et al., 2013; van der Vorst et al., 1998).

Products with age dependent deterioration rates have insufficient quality as the main registered cause of food waste. This is expected as most of the products do not have due dates to be judged by. Many of the underlying causes are, however, similar as for products with fixed shelf life. An additional planning challenge for products with age dependent deterioration rates is considering their quantity and quality levels, and remaining shelf life of such products in the planning decisions. This is mainly due to missing technology that would allow visibility of such information and that would utilize them to increase the accuracy of future demand or safety stocks. Better utilization of barcodes or RFID (radio frequency identification) technology for capturing additional data about products in the ERP system could reduce food waste.

Typical handling causes of food waste discovered in the warehouse are damages caused by the operators when picking the products or transporting them to the storage. Additionally, pallets with temperature sensitive products are sometimes kept waiting in the area with inappropriate temperature levels. Alternative layout organization or training of operators could improve the physical handling of such products.

Transportation food waste created for both products with fixed shelf life and age dependent deterioration rates is mainly due to inappropriate handling during the loading and unloading process or during the transportation. Among the main challenges is the organization of pallets inside the trucks with frozen and chilled temperature zones, mostly organized based on the order of the stores. Temperature sensitive products placed against the isolated partition panel between two temperature zones, or against an outer wall whilst outside temperatures are very low might be exposed to either too low or high temperatures, thus reducing quality level. This is an even bigger challenge for deliveries to distant stores with longer delivery times. Including information about product sensitivity in route planning could identify optimal trade-offs between space allocation and potential level of food waste created in different scenarios.

Pre-store rejections occur mainly due to insufficient quality (products with age dependent deterioration rate) or short remaining shelf life (products with fixed shelf life). The underlying causes lie at the wholesaler warehouse. For example, time and quality decrease for products that are picked at the stock of the wholesaler and kept in the temporary waiting area before they are dispatched to stores. The temperature in the temporary area is either higher or lower than is required by the products and the time spent in this area sometimes exceeds two days. This information is, however, not considered when planning the picking orders and deliveries to stores. Pre-store rejections could be thus reduced by considering the shelf life, waiting time and temperature information during the planning and control of deliveries in the warehouse. This would consequently lead also to saving additional transportation and disposal costs.

In-store food waste of products with fixed shelf life and products with age dependent deterioration rate is created due to similar reasons. From a planning perspective it is lack of data captured and utilized in replenishment process that causes the most 
problems. Information about current volumes or expiration dates of products in shelves are checked only manually without using suitable information technology that captures and utilizes the mentioned data. Forecasts and safety stock levels are therefore calculated only based on an experience of planners who sometimes consider historical sales. High order and delivery frequency allow high turnover stores to plan without very precise forecast. For the smaller stores higher ordering frequency and smaller batch sizes could reduce food. Capturing and utilizing data about the current product volumes and remaining shelf life of the products in the shelves could increase visibility of the products, and reduce uncertainty and food waste. Other causes of instore food waste have been indicated mechanical damages caused by customers or operators. Utilizing equipment ensuring the right temperature and display of the products in the shopping area could lead to reduction of mechanical damages in the stores.

The above discussed causes are summarized and grouped in the fishbone diagram under four areas, namely planning decisions, data utilization, execution of plan and damaged products, in order to highlight the main problematic areas (see Fig. 3).

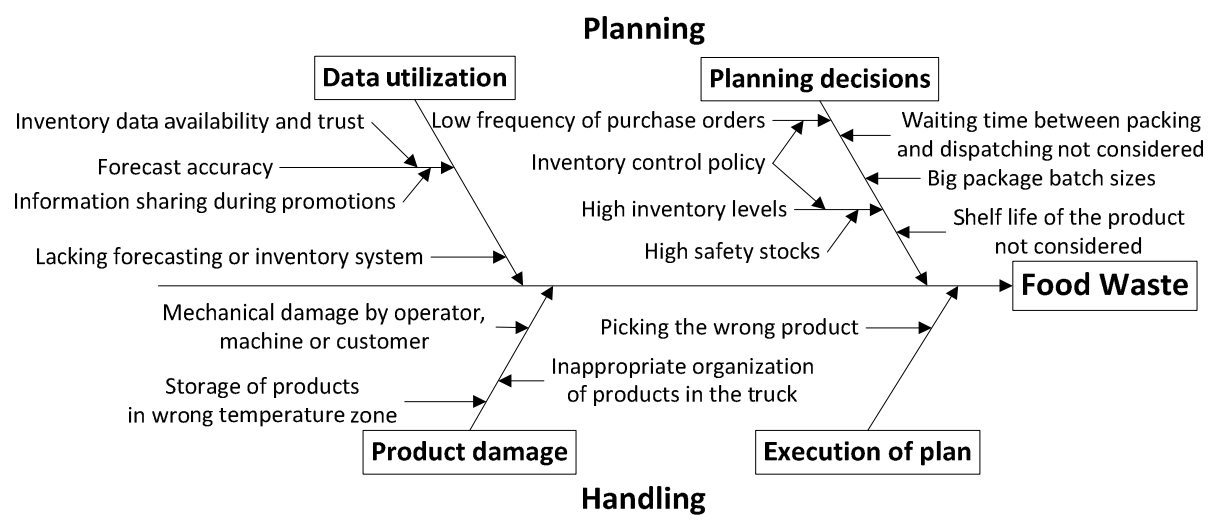

Fig. 3. Planning and handling causes of food waste

\section{Conclusion}

The study presents the main and underlying causes of food waste for products with fixed shelf life and age dependent deterioration rates as identified in the case companies. The underlying causes of food waste are structured into four areas, namely planning decisions, data utilization, execution of plans, and damaged products. More research is necessary to validate the findings of this study. Some of the identified causes might not be that relevant for other countries. For example, companies in other countries might be able to cope with low delivery frequency or big batch sizes better than companies in Norway, as they do not face the challenges such as long delivery lead times or long distances traveled due to fjords, ferries or temporary closed roads during the winter period. Future research could focus on deeper analysis and quantification of identified causes in order to understand the relative impact of individual 
causes on food waste and so to better identify improvement options. The main focus could be on quantifiable causes of food waste as forecasting accuracy, inventory and safety stock levels and promotions which have been highlighted also by other researchers. Furthermore, trade-offs between food waste and availability should be investigated and discussed. The study contributes to the literature by in-depth analysis of quantitative and qualitative data on planning and handling causes of food waste in the FDC which is still rare in the current research. Practitioners in a similar industry may use the findings as a baseline for identification of food waste causes in their companies.

Acknowledgement. Authors of the paper would like to express a deep gratitude to the case companies involved, to the researchers Peter Falster and Chris Martin, and to research organization NordForsk which funds the LogiNord project.

\section{References}

1. APICS (ed.): APICS Dictionary - The essential supply chain reference, 14th edn. (2013)

2. Bakker, M., Riezebos, J., Teunter, R.H.: Review of inventory systems with deterioration since 2001. European Journal of Operational Research 221(2), 275-284 (2012)

3. Beretta, C., Stoessel, F., Baier, U., Hellweg, S.: Quantifying food losses and the potential for reduction in Switzerland. Waste Management (2012)

4. Eriksson, M., Strid, I., Hansson, P.A.: Food losses in six Swedish retail stores: Wastage of fruit and vegetables in relation to quantities delivered. Resources, Conservation and Recycling 68, 14-20 (2012)

5. Garrone, P., Melacini, M., Perego, A.: Opening the black box of food waste reduction. Food Policy 46, 129-139 (2014)

6. Gustavsson, J., Cederberg, C., Sonesson, U., van Otterdijk, R., Meybeck, A.: Global food losses and food waste. FAO of the United Nations, Rom (2011)

7. Hanssen, O.J., Møller, H.: Food Wastage in Norway (2013)

8. Kaipia, R., Dukovska-Popovska, I., Loikkanen, L.: Creating sustainable fresh food supply chains through waste reduction. International Journal of Physical Distribution \& Logistics Management 43(3), 262-276 (2013)

9. Mena, C., Adenso-Diaz, B., Yurt, O.: The causes of food waste in the supplier-retailer interface: Evidences from the UK and Spain. Resources, Conservation and Recycling 55(6), 648-658 (2011)

10. Muller, S.: Food waste in Norway: Root causes analysis at the producer - retailer stage (Master Thesis). BI Norwegian Business School, Oslo (2013)

11. Romsdal, A., Thomassen, M., Dreyer, H.C., Strandhagen, J.O.: Fresh food supply chains; characteristics and supply chain requirements. Paper Presented at the 18th International Annual EurOMA Conference, Cambridge, UK (2011)

12. Shapiro, R.D., Heskett, J.L.: Logistics Strategy: cases and concepts. West Publishing Company St., Paul (1985)

13. Stevenson, W.J., Hojati, M.: Operations management, vol. 8. McGraw-Hill/Irwin, Boston (2007)

14. Taylor, D.H., Fearne, A.: Demand management in fresh food value chains: A framework for analysis and improvement. Supply Chain Management 14(5), 379-392 (2009)

15. van der Vorst, J.G.A.J., Beulens, A.J.M., de Wit, W., van Beek, P.: Supply Chain Management in Food Chains: Improving Performance by Reducing Uncertainty. International Transactions in Operational Research 5(6), 487-499 (1998)

16. Yin, R.K.: Case study research: Design and methods, vol. 5. Sage Publications, Inc. (2009) 